\title{
Article \\ Juvenile Hormone Synthesis Pathway Gene SfIPPI Regulates Sogatella furcifera Reproduction
}

\author{
Ming-Fu Gong ${ }^{1,+}{ }^{+}$, Xi-Bin Yang ${ }^{1}$, Gui-Yun Long ${ }^{1} \oplus$, Ze-Yan Jia ${ }^{1}$, Qing-Hui Zeng ${ }^{1}$, Dao-Chao Jin ${ }^{1}$, Hong Yang ${ }^{1, *}$ \\ and Cao Zhou ${ }^{2,+}$ \\ 1 Provincial Key Laboratory for Agricultural Pest Management of Mountainous Region, \\ Institute of Entomology, Guizhou University, Guiyang 550025, China; gongmingfu2019@126.com (M.-F.G.); \\ yangxibingz@126.com (X.-B.Y.); lgy0256@126.com (G.-Y.L.); Jiazeyan520@163.com (Z.-Y.J.); \\ 18311516405@163.com (Q.-H.Z.); daochaojin@126.com (D.-C.J.) \\ 2 College of Life Sciences, Chongqing Normal University, Chongqing 401331, China; zhouc@cqnu.edu.cn \\ * Correspondence: axyridis@163.com \\ + These authors contributed equally to this work.
}

Citation: Gong, M.-F.; Yang, X.-B.; Long, G.-Y.; Jia, Z.-Y.; Zeng, Q.-H.; Jin, D.-C.; Yang, H.; Zhou, C. Juvenile Hormone Synthesis Pathway Gene SfIPPI Regulates Sogatella furcifera Reproduction. Insects 2022, 13, 174. https://doi.org/10.3390/ insects13020174

Academic Editor: Lynn M. Riddiford

Received: 20 December 2021

Accepted: 31 January 2022

Published: 6 February 2022

Publisher's Note: MDPI stays neutral with regard to jurisdictional claims in published maps and institutional affiliations.

Copyright: (C) 2022 by the authors. Licensee MDPI, Basel, Switzerland. This article is an open access article distributed under the terms and conditions of the Creative Commons Attribution (CC BY) license (https:// creativecommons.org/licenses/by/ $4.0 /)$.
Simple Summary: The juvenile hormone is essential for insect growth, development, and reproduction. Isopentenyl pyrophosphate isomerase is a key isomerase involved in the synthesis of the juvenile hormone. This study evaluates the effect of the targeted silencing of the SfIPPI gene on the reproduction of Sogatella furcifera (white-backed planthopper). We found that SfIPPI silencing significantly inhibits the ovarian development and egg production in female adults of $S$. furcifera and significantly inhibits the transcription of downstream genes in the juvenile hormone synthesis pathway. Our data provide insight into the function of juvenile hormone biosynthetic pathway genes in insect reproduction, which could be a potential target to control and prevent agricultural pests.

\begin{abstract}
The juvenile hormone $(\mathrm{JH})$ is crucial for insect reproduction, and isopentenyl pyrophosphate isomerase (IPPI) is a key enzyme in the JH synthesis pathway. However, few studies have investigated how IPPI regulates insect reproduction. This study identifies and characterizes the IPPI gene (SfIPPI) from the important agricultural pest Sogatella furcifera. A phylogenetic analysis reveals a high homology of SfIPPI with the IPPI amino acid sequences of Laodelphax striatellus and Nilaparvata lugens (Stål). Furthermore, SfIPPI is expressed at various developmental stages and in various tissues of S. furcifera, and is significantly higher on the 5th day of adult emergence and in integument tissue, while lower levels are found on the 3rd day of adult emergence and in fat body and gut tissue. After silencing SfIPPI using RNA interference, the ovarian development is significantly inhibited and the fecundity is significantly reduced when compared with the control group. Additionally, SfIPPI silencing significantly decreases the expression levels of downstream JH signal transduction pathway genes (SfJHAMT, SfFAMeT, and $S f K r-h 1$ ) and $S f V g$. Our findings are helpful in elucidating the molecular mechanism underlying the regulation of insect reproduction through genes in the JH synthesis pathway, and they provide a theoretical basis for the development of pest control treatments targeting SfIPPI.
\end{abstract}

Keywords: Sogatella furcifera; juvenile hormone; synthesis pathway; isopentenyl pyrophosphate isomerase (IPPI); RNA interference; reproduction

\section{Introduction}

The juvenile hormone $(\mathrm{JH})$ is a sesquiterpenoid compound synthesized in the corpora allata of insects [1,2]. It is essential for regulating the physiological processes of insect growth, metamorphosis, reproduction, and immunity [3-8]. JH biosynthesis is associated with the expression of most JH biosynthetic enzymes in the corpora allata [9]. Isopentenyl pyrophosphate isomerase (IPPI) is an isomerase involved in JH synthesis $[10,11]$ that catalyzes the conversion of isopentenyl pyrophosphate (IPP) to dimethylpropylene 
pyrophosphate (DMAPP). IPPIs were first extracted from the lepidopteran insect Bombyx mori in 1985 [12], and the IPPI sequence was, subsequently, cloned from insects such as Choristoneura fumiferana and Manduca sexta [13]. Later, a study of the molecular characteristics and function of Aedes aegypti AaIPPI revealed that it was important for JH synthesis because changes in the level of AaIPPI mRNA affected JH biosynthesis [14]. However, there are relatively few studies investigating how IPPIs regulate insect reproduction.

The white-backed planthopper, Sogatella furcifera (Horváth) (Hemiptera: Delphacidae), is an important insect pest in rice production in China and Asia [15]. S. furcifera completes its generational development with the use of rice crops and weeds, causing extensive crop damage. It is characterized by a short life cycle, long-distance migration, and strong reproductive and environmental adaptability [16,17]. Most of the crop damage is caused by adults and nymphs directly sucking the phloem sap of rice plants and by females laying eggs. S. furcifera is also a transmission vector for the southern rice black-streaked dwarf virus [18], which negatively affects rice production and leads to severe economic losses [19-21]. At present, S. furcifera is mainly controlled using chemical insecticides, but their excessive use not only negatively impacts the environment and natural enemies of $S$. furcifera, but also lead to insecticide resistance and other problems [22-24]. Thus, to achieve a sustainable pest management, it is imperative to find an environmentally friendly and efficient method to control $S$. furcifera populations.

Insects have a strong reproductive ability, and studies of the regulation of their reproductive mechanisms are crucial for pest control. Significant work has been conducted on the role of the $\mathrm{JH}$ in the regulation of reproduction in the brown planthopper, N. lugens. Previous studies have shown that $\mathrm{JH}$ analogues can stimulate vitellogenesis in this species [25]. An adequate nutrition has been found to affect JH biosynthesis and, thus, reproductive maturation [26,27]. Additionally, the loss of brummer-mediated lipolysis was found to impair vitellogenesis and oocyte maturation by working through the $\mathrm{JH}$ signaling pathway [28]. In this study, we clone and identify SfIPPI based on the published genome [29] and transcriptome data [30] of S. furcifera. We used quantitative (q) polymerase chain reaction (PCR) assays to detect the expression levels of SfIPPI in different developmental stages and tissues, and also used RNA interference (RNAi) technology to study the function of this gene in $S$. furcifera reproduction. Our findings provide a reference for the sustainable management of $S$. furcifera by identifying suitable target genes to control the rapid propagation of these pests.

\section{Materials and Methods}

\subsection{Insect Rearing and Collection}

S. furcifera were collected from a rice field in Huaxi, Guiyang, Guizhou, China, in 2013. They were normally reared and reproduced on Taichung Native 1 rice in our laboratory under specific environmental conditions (temperature, $25 \pm 1{ }^{\circ} \mathrm{C}$; relative humidity $70 \% \pm 5 \%$; photoperiod, $16 \mathrm{~h}$ light: $8 \mathrm{~h}$ dark). They were kept in an isolated area so that the adults could be used as the source of test insects.

\subsection{Sample Preparation}

We collected and prepared samples of adult $S$. furcifera to determine the expression levels of SfIPPI in different developmental stages and tissues. Firstly, we paired and reared newly emerged females and males of $S$. furcifera in test tubes with rice seedlings that were replaced daily. During the feeding process, we randomly collected samples from egg to adult at 16 time points: egg $(n=40) ; 1$ st $(n=40)$; 2 nd $(n=40)$; 3rd $(n=35)$; 4 th at $1 \mathrm{~d}$ and $2 \mathrm{~d}(n=30) ; 5$ th at $1 \mathrm{~d}, 2 \mathrm{~d}$, and $3 \mathrm{~d}(n=20)$ and female adults at $12 \mathrm{~h}, 1 \mathrm{~d}, 2 \mathrm{~d}$, $3 \mathrm{~d}, 4 \mathrm{~d}$, and $5 \mathrm{~d}$ after eclosion $(n=15)(n=15-40$ individuals per replicate, respectively). Secondly, we dissected the head $(n=50)$, gut $(n=100)$, fat body $(n=50)$, ovary $(n=30)$, and integument $(n=100)$ tissues from $24 \mathrm{~h}$ old female adults in phosphate-buffered saline (PBS; $\mathrm{pH}=7.4$ ). All samples were subjected to three biological replicates. Each collected 
sample was immediately frozen in liquid nitrogen and placed in a $1.5 \mathrm{~mL}$ RNAse-free microcentrifuge tube before storing at $-80^{\circ} \mathrm{C}$ until RNA extraction.

\subsection{Total RNA Extraction and $c D N A$ Synthesis}

We extracted total RNA from samples collected at different developmental stages and tissues of $S$. furcifera using an EZNA HP Total RNA Kit (Omega Bio-tek, Norcross, GA, USA) according to the manufacturer's recommendations. The concentration of the extracted RNA was determined using a NanoDrop 2000 spectrophotometer (Thermo Fisher Scientific, Waltham, MA, USA). The RNA integrity was evaluated following $1 \%$ agarose gel electrophoresis. Then, the extracted qualified RNA underwent reverse transcription using a PrimeScript RT Reagent Kit with gDNA Eraser (TaKaRa, Dalian, China) to synthesize first-strand cDNA, which was stored at $-20{ }^{\circ} \mathrm{C}$ until use.

\subsection{SfIPPI Cloning}

Based on the published genome [29] and transcriptome [30] data of S. furcifera, we searched for the cDNA sequence of SfIPPI using the BLAST tool (https:/ /blast.ncbi.nlm. nih.gov / Blast.cgi, accessed on 26 May 2021) on the NCBI website. Following alignment of the obtained sequences, we designed primers for the open reading frame (ORF) sequence of SfIPPI (Table 1) using Primer Premier 6.0 software (PREMIER Biosoft International, Palo Alto, CA, USA). Then, we performed PCR assays using the first-strand cDNA that we already obtained by reverse transcription as the template. The PCR reaction mixture included $12.5 \mu \mathrm{L}$ PCR master mix, $1 \mu \mathrm{L}$ each of the forward and reverse primers, and $3 \mu \mathrm{L}$ S. furcifera cDNA template, which was composed to a final volume of $25 \mu \mathrm{L}$ using $\mathrm{ddH}_{2} \mathrm{O}$. The cycling conditions were as follows: pre-denaturation at $94{ }^{\circ} \mathrm{C}$ for $3 \mathrm{~min}$; 30 cycles of denaturation at $94{ }^{\circ} \mathrm{C}$ for $30 \mathrm{~s}$, annealing at $55^{\circ} \mathrm{C}$ for $30 \mathrm{~s}$, and extension at $72{ }^{\circ} \mathrm{C}$ for $1 \mathrm{~min}$; final extension at $72{ }^{\circ} \mathrm{C}$ for $10 \mathrm{~min}$. The PCR products were stored at $4{ }^{\circ} \mathrm{C}$ and evaluated following $1 \%$ agarose gel electrophoresis. Then, the target band was excised from the gel and purified using an EasyPure Quick Gel Extraction Kit (Quanshijin Biotechnology, Beijing, China) according to the manufacturer's instructions. The recovered PCR product was ligated to a pMD18-T vector (TaKaRa, Dalian, China) and transformed into competent Escherichia coli DH5 $\alpha$ cells (Quanshijin Biotechnology, Beijing, China). Finally, possible colonies containing the vector were sent to Sangon Biotech (Shanghai, China) for sequencing.

\subsection{Sequencing Analysis}

After using SeqMan software (DNASTAR, Madison, WI, USA) to assemble and proofread the sequencing data, we used DNAMAN 7.0 software (Lynnon Biosoft, San Ramon, CA, USA) to deduce the amino acid sequence. We also used the BLAST tool on the NCBI website to further confirm the target gene and obtain the homologous sequences. We used the ORF Finder tool (https://www.ncbi.nlm.nih.gov/orffinder/, accessed on 27 May 2021) on the NCBI website to identify the ORFs of SfIPPI; ProtParam (https://web.Expasy.org/protparam/, accessed on 27 May 2021) to predict the amino acid molecular composition, relative molecular mass, isoelectric point, and other physical and chemical properties of the encoded protein; the SMART database (http: //smart.embl-heidelberg.de/, accessed on 27 May 2021) to predict conserved domains. We constructed a phylogenetic tree using the neighbor-joining method in MEGA 6.06 software [31] with 1000 bootstrap replications.

\subsection{SfIPPI Expression Different Developmental Stages and Tissues}

We used Primer Premier 6.0 software to design qPCR primers (Table 1) based on the SfIPPI sequence obtained from the previous cloning and identification experiments. qPCR analysis was performed on a CFX96 Real-Time PCR System (Bio-Rad, Hercules, CA, USA) using FastStart Essential DNA Green Master hot-start reaction mix (Roche, Indianapolis, IN, USA) to detect the expression levels of SfIPPI in the different developmental stages 
and tissues of $S$. furcifera. The qPCR reaction was performed in a final volume of $20 \mu \mathrm{L}$ containing $1 \mu \mathrm{L}$ sample cDNA, $1 \mu \mathrm{L}$ of each forward and reverse primer $(10 \mu \mathrm{M}), 7 \mu \mathrm{L}$ RNase-free water, and $10 \mu \mathrm{L}$ FastStart Essential DNA Green Master. The qPCR cycling parameters were initially denatured at $95^{\circ} \mathrm{C}$ for $10 \mathrm{~min}$, followed by 40 cycles of amplification $\left(95^{\circ} \mathrm{C}\right.$ for $30 \mathrm{~s}$ and $60{ }^{\circ} \mathrm{C}$ for $\left.30 \mathrm{~s}\right)$. Melting curve analysis was performed from $65{ }^{\circ} \mathrm{C}$ to $95^{\circ} \mathrm{C}$. The ribosomal protein L9 (GenBank accession number: KM885285) and $\alpha-1$ tubulin (GenBank accession number: KP735521) genes were used as internal controls. Three biological replicates and three technical replicates were performed for each developmental stage and tissue.

Table 1. Primers used in this research.

\begin{tabular}{|c|c|c|}
\hline Experiment & Primer & Primer Sequence $\left(5^{\prime}\right.$ to $\left.3^{\prime}\right)$ \\
\hline \multirow{2}{*}{ cDNA cloning } & IPPI-i-F & ATTTGGTAGCAGAGCCATAAGA \\
\hline & IPPI-i-R & CTCCTGGTTCGCTTCAATT \\
\hline \multirow{18}{*}{ qPCR } & Y-IPPI-F & GCCTGTTGCAGTCATCCGTTGT \\
\hline & Y-IPPI-R & GCGGTATGCCTAGCTCGTGGTT \\
\hline & $\mathrm{Y}-\mathrm{Vg}-\mathrm{F}$ & AGTGGTGAGGTGCGTGGTCT \\
\hline & Y-Vg-R & CGTTGCTGCTGCTACCTGACA \\
\hline & Y-VgR-F & CTGCGAACACAGCCGAATGGA \\
\hline & Y-VgR-R & GGAACTGCGACTGCGTATCACA \\
\hline & Y-JHAMT-F & ACGAGAACCGTAATGGCAGTCA \\
\hline & Y-JHAMT-R & CCAGGACCACATCCAACATCCA \\
\hline & Y-FAMeT-F & CTCTTGAACTGACGACCGAGGA \\
\hline & Y-FAMeT-R & CGACCAGCCGCCTATGAAGAT \\
\hline & Y-Met-F & GCCGCCAGTTGACCGATTACA \\
\hline & Y-Met-R & ACCAGCAGAGTCGCACGAGT \\
\hline & Y-Kr-h1-F & CTCACCGCAGCACTCAACTCA \\
\hline & Y-Kr-h1-R & AGGCACAGGCGACATTAGAACA \\
\hline & Y-RPL9-F & GGGCGAGAAGTACATCCGTAGG \\
\hline & Y-RPL9-R & GCGGCTGATCGTGAGACATCTT \\
\hline & Y-TUB-F & CGCTGTTGATGGAGAGGCTGTC \\
\hline & Y-TUB-R & ACGACGGCTGTGGATACCTGTG \\
\hline \multirow{4}{*}{ dsRNA synthesis } & T7-IPPI-F & TAATACGACTCACTATAGGGTAGCAGAGCCATAAGAAGTT \\
\hline & T7-IPPI-R & TAATACGACTCACTATAGGGGCAGGATTAGAATGTAGTCG \\
\hline & T7-GFP-F & TAATACGACTCACTATAGGGGCCAACACTTGTCACTACTT \\
\hline & T7-GFP-R & TAATACGACTCACTATAGGGGGAGTATTTTGTTGATAATGGTCG \\
\hline
\end{tabular}

Note: Underlined nucleotides indicate DNA sequences transcribed downstream of the T7 promoter. RT-qPCR reverse transcription real-time polymerase chain reaction; ds RNA, double-stranded RNA.

\subsection{Double-Stranded RNA Synthesis}

Based on the previously obtained SfIPPI sequence, we designed gene-specific primers containing the T7 polymerase promoter sequence at the $5^{\prime}$-terminal using the E-RNAi website, an online tool for the design and evaluation of RNAi reagents. (https:/ / www.dkfz. de/signaling/e-rnai3/, accessed on 12 June 2021) (Table 1). Then, we used these primers for PCR amplification of our previously synthesized first-strand cDNA to generate a DNA template containing the T7 promoter sequence. Next, we used TA cloning to expand and cultivate the population of transformed bacteria in a liquid medium. Then, the plasmid was extracted, and the high-concentration gel-recovered dsDNA was used as the template for dsRNA synthesis. The dsRNA was synthesized using a TranscriptAid T7 High-Yield Transcription Kit and GeneJET RNA Purification Kit (both Thermo Fisher Scientific) according to the manufacturer's instructions. The concentration of the dsRNA was determined using a NanoDrop 2000 spectrophotometer, and its integrity was evaluated following 1\% agarose gel electrophoresis. At the same time, the green fluorescent protein (GFP) gene was used to synthesize GFP dsRNA by the same method for use in the control group. 


\subsection{RNAi Experiment}

We performed an RNAi experiment to investigate the function of SfIPPI in S. furcifera reproduction. Newly emerged (1-12 h) female adults of $S$. furcifera were selected as the test insects in this study. Following anesthetization with $\mathrm{CO}_{2}$ for $30 \mathrm{~s}$, we placed the test insects in empty culture plates. Then, we used an IM-31 microinjector (NARISHIGE, Tokyo, Japan) to inject $100 \mathrm{~nL}$ dsIPPI into the thorax between the middle and hind leg. An equal volume of dsGFP was injected in the negative control group. Three biological replicates were performed for each treatment, with 100 female adult injections for each replicate. The injected insects were placed in a test tube containing fresh rice seedlings and kept in an artificial climate chamber (temperature; $25 \pm 1{ }^{\circ} \mathrm{C}$; relative humidity, $70 \pm 5 \%$, and photoperiod, $16 \mathrm{~h}$ light: $8 \mathrm{~h}$ dark) for $48 \mathrm{~h}$. Then, 10 of the surviving S. furcifera were randomly selected from each experimental group to determine RNAi efficiency using reverse transcription (RT)-qPCR. At the same time, the transcription levels of $S f V g$ and $S f V g R$ genes and $\mathrm{JH}$ synthesis pathway-related genes were determined to clarify their regulatory relationship. Three biological replicates were performed in this experiment, and 10 injected adults were used for each biological replicate.

\subsection{Bioassay}

To assess the effect of RNAi on the fertility of $S$. furcifera, we injected emerged females with dsIPPI or dsGFP (as the control). Then, one female and two males (two uninjected males, excluding the influence of males on mating) were paired after injection in glass tubes containing fresh rice seedlings. Each treatment group contained 15 insect parings, and three biological replicates were performed. Fresh rice seedlings were provided every 2 days, and the old seedlings were kept in the tube for further culture and observation. The number of newly hatched nymphs was recorded daily. After 10 days, the old rice seedlings were dissected under a stereomicroscope, and the number of hatched nymphs and unhatched eggs were also counted until the female adults died. Ovaries were dissected 6 days after injection and washed with PBS before using a stereoscopic microscope (SMZ25 Nikon Corporation, Tokyo, Japan) to observe, compare, and photograph ovarian development.

\subsection{Statistical Analysis}

The $2^{-\Delta \Delta C t}$ method [32] was used to calculate the relative expression of SfIPPI and other genes in the JH synthesis pathway. All experimental data were analyzed using SPSS 22.0 statistical software (IBM Corp., Armonk, NY, USA). Significant differences between the treatments were assessed using one-way analysis of variance, followed by the Tukey test for multiple comparisons. The Student $t$-test for independent samples was used to analyze the significance of the efficiency of RNAi silencing.

\section{Results}

\subsection{Sequence Identification and Characteristics of SfIPPI}

According to the verification results, the final full-length ORF of SfIPPI from S. furcifera contained $633 \mathrm{bp}$ that encoded a hypothetical protein sequence of 210 amino acids. After BLAST alignment, we named it SfIPPI (GenBank accession number: OM417142) according to its sequence similarity and conserved domains. The online software ProtParam predicted the molecular formula of the S. furcifera IPPI protein as $\mathrm{C}_{1090} \mathrm{H}_{1680} \mathrm{~N}_{310} \mathrm{O}_{312} \mathrm{~S}_{9}$ with a molecular weight of approximately $24.41 \mathrm{kDa}$ and a theoretical isoelectric point of 6.30. The conserved domain analysis revealed that the protein contained a typical conserved domain of the Nudix family (amino acid positions 71-102), a conserved cysteine and conserved glutamic acid motifs, and seven conserved domains that are key to the catalytic activity of IPPI enzymes (Figure 1). 


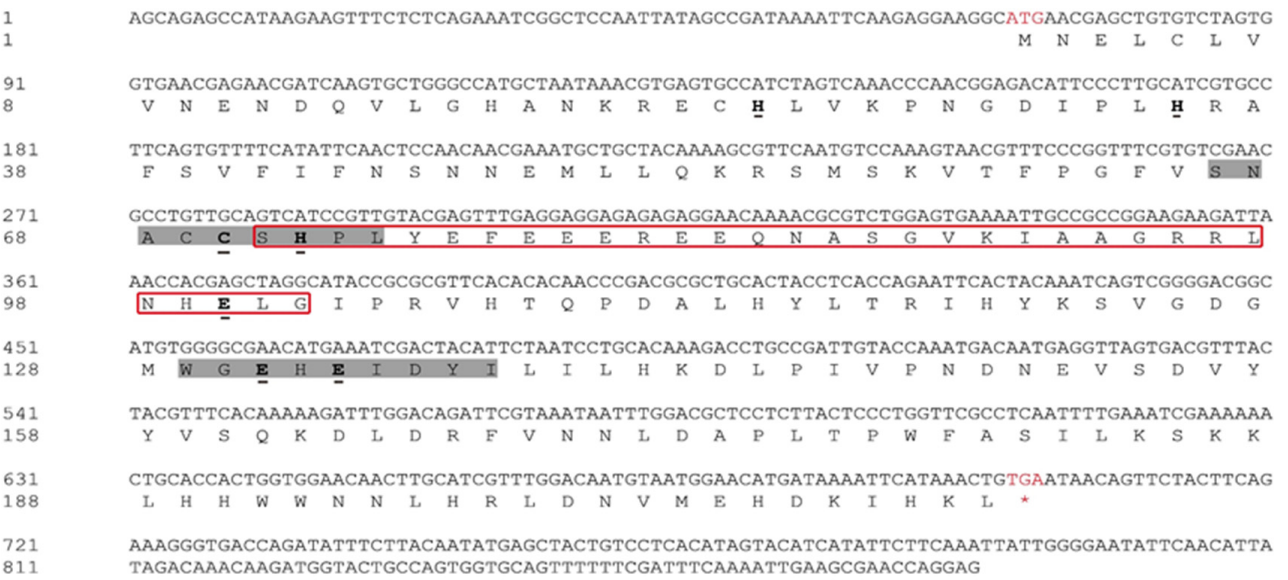

Figure 1. Nucleotide and amino acid sequence analysis of SfIPPI in S. furcifera. Red font ATG, start codon; red font TGA with an asterisk, stop codon; red box, Nudix hydrolase domain; bold and underlined amino acids, residues critical for the catalytic activity of the enzyme; amino acids with a shaded background, conserved cysteine and conserved glutamic acid motifs.

\subsection{Sequence Comparisons and Phylogenetic Analysis}

We investigated the degree of similarity between the IPPI protein of $S$. furcifera and that of other species using a BLAST homology search and comparison analysis. SfIPPI had high similarities of $92.38 \%$ and $85.24 \%$ with Laodelphax striatellus (GenBank accession number: RZF47560) and Nilaparvata lugens (Stål) (GenBank accession number: XP_022187401), respectively. Furthermore, there was a 57.14\% similarity with the hemipteran Halyomorpha halys (GenBank accession number: XP_014271459). The phylogenetic tree (Figure 2) showed the $S$. furcifera IPPI protein clustered with N. lugens, L. striatellus, H. halys, and Riptortus pedestris, forming a branch comprising Hemiptera. However, the S. furcifera IPPI protein was most closely related to that of $N$. lugens and L. striatellus.

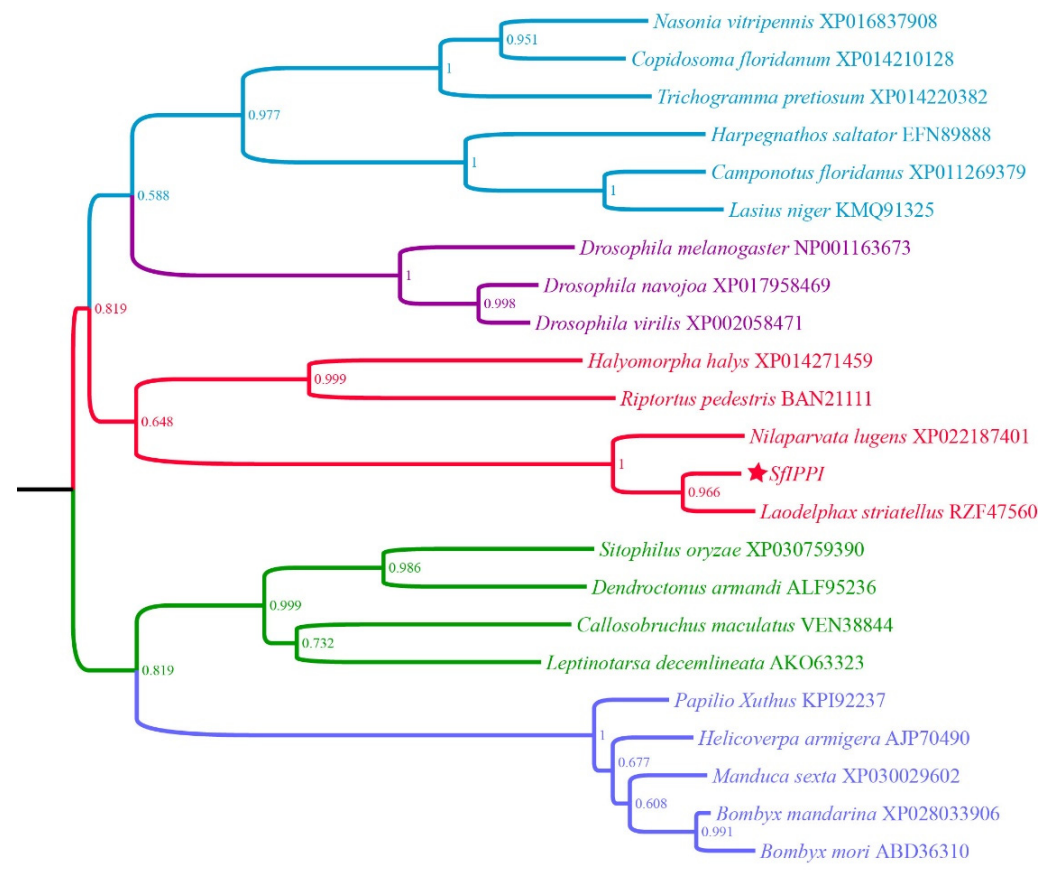

0.04

Figure 2. Phylogenetic analysis of SfIPPI homologs from insect species based on amino acid sequences. Sequences were downloaded from the GenBank protein database. The red star indicates the IPPI gene of $S$. furcifera. 


\subsection{SfIPPI Expression in Different Developmental Stages and Tissues}

The expression pattern of SfIPPI in the different developmental stages of S. furcifera was detected by qPCR. SfIPPI was expressed and fluctuated in all developmental stages of S. furcifera. The expression level was higher in the third instar and fourth instar $1 \mathrm{~d}$ old nymphs and in emerged female adults at $12 \mathrm{~h}, 1 \mathrm{~d}$, and $5 \mathrm{~d}$. The lowest expression level was in $3 \mathrm{~d}$ old female adults (Figure 3A). SfIPPI was expressed in various tissues of S. furcifera adults. The levels were highest in the integument and head, followed by the ovary, and lowest in the gut and fat body, with the fat body showing the lowest level of expression (Figure 3B).
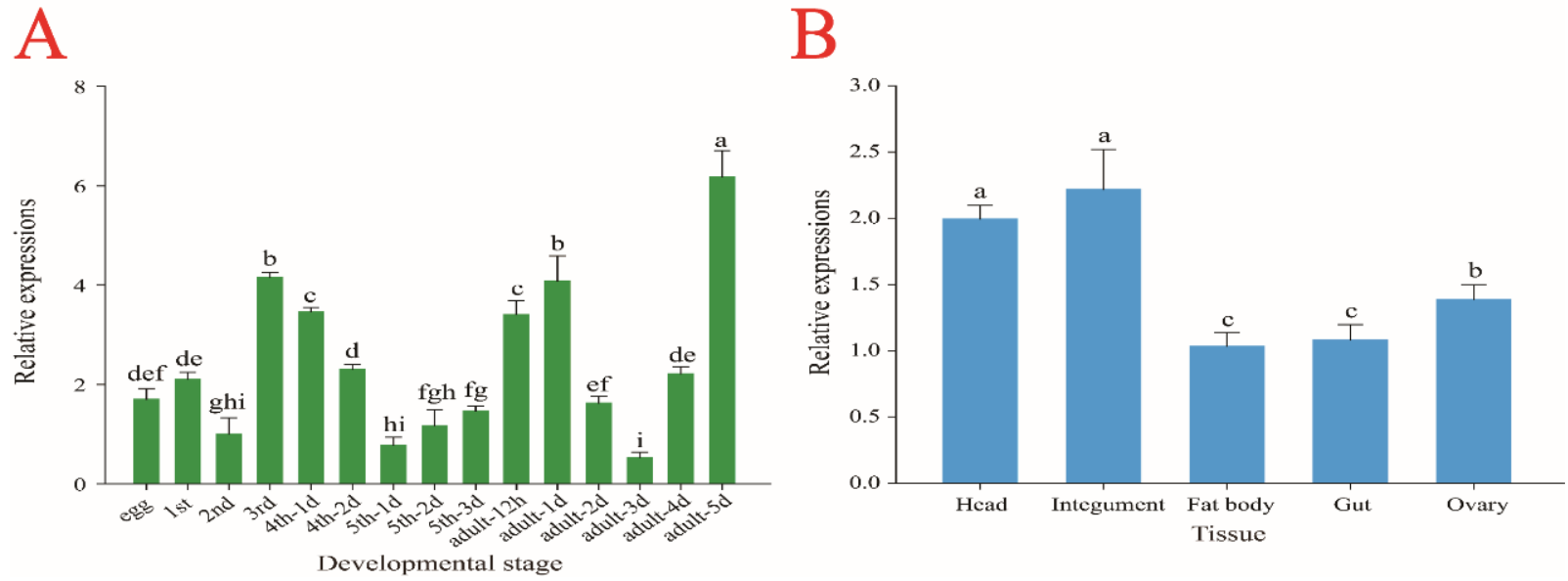

Figure 3. Relative expression levels of SfIPPI in the different development stages and tissues of $S$. furcifera. SfRPL9 and SfTUB were used as internal control genes. (A) Relative expression levels of SfIPPI from egg to $5 \mathrm{~d}$ old adult as determined by qPCR. (B) Relative expression levels of SfIPPI in different tissues of $24 \mathrm{~h}$ old female adults as determined by qPCR. The results were expressed as the mean \pm standard error of the mean of three biological replicates and their respective three technical replicates. Different lowercase letters above bars indicate significant differences in the gene expression level between different developmental stages and tissues $(p<0.05$, Tukey).

\subsection{Effect of Silencing of SfIPPI on Reproduction of S. furcifera Female Adults}

We determined the level of SfIPPI mRNA $48 \mathrm{~h}$ after injecting newly emerged females with dsIPPI of $S$. furcifera. Compared with the control group (injected with dsGFP), the expression of SfIPPI was significantly suppressed and downregulated by $66.35 \%$ (Figure $4 \mathrm{~A}$ ), indicating that SfIPPI was successfully silenced. Furthermore, the number of females laying eggs was reduced to $73.37 \%$ in the dsIPPI group, which was significantly lower than the control group (219 eggs) (Figure 4B), indicating that SfIPPI significantly affected the number of eggs laid by S. furcifera. To further understand the effect of SfIPPI on the ovarian development of $S$. furcifera, we dissected the ovaries $6 \mathrm{~d}$ after injection to observe their development (Figure 4C). We observed a large number of typical banana-shaped mature oocytes in the dsGFP group, indicating that dsGFP injection did not affect ovarian development. However, a few mature oocytes were observed in the dsIPPI treatment group, suggesting that SfIPPI significantly affected ovarian development.

\subsection{Effect SfIPPI Silencing on Other Genes in the JH Signal Transduction Pathway}

It has been reported that the $\mathrm{JH}$ signaling pathway gene and vitellogenin gene can regulate insect reproduction. In order to clarify the mechanism of the effect of SfIPPI gene silencing on the reproduction of $S$. furcifera, we detected changes in the expression of genes related to the $\mathrm{JH}$ signal transduction pathway of $S$. furcifera in newly emerged females, following injection with dsIPPI. The expression levels of SfJHAMT and SfFAMeT and those of $S f K r-h 1$ and $S f V g$ were significantly lower compared to the control group $(p<0.05$ and $p<0.01$, respectively). The expression levels of SfMet and SfVgR did not change significantly (Figure 5). 

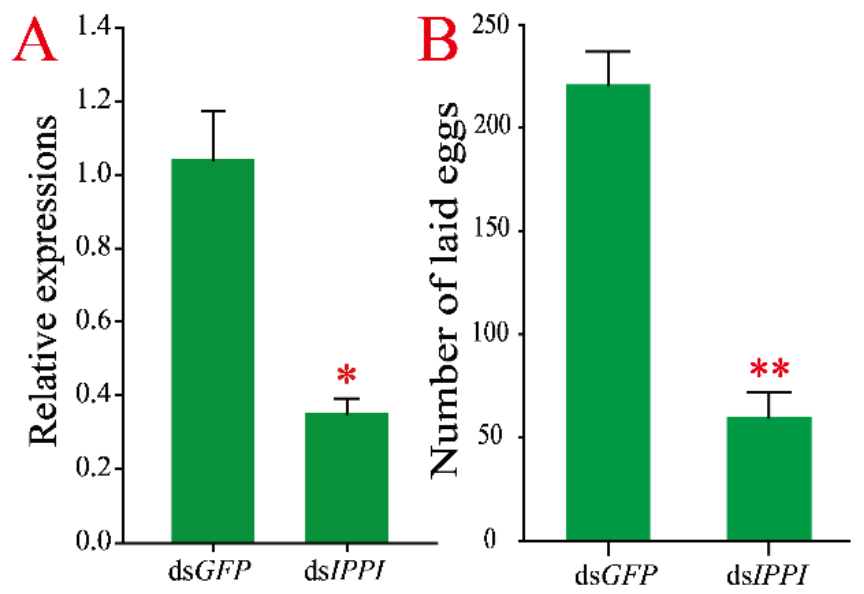

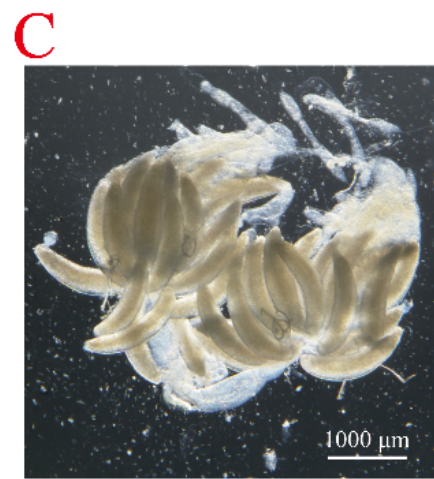

ds $G F P$

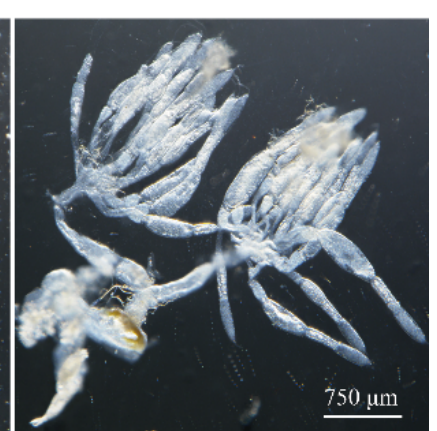

$\mathrm{ds} I P P I$

Figure 4. Comparisons of the S. furcifera treatment group (dsIPPI) and control group (dsGFP). (A) Effects of SfIPPI silencing at the transcription level; (B) effects of SfIPPI silencing on reproduction; (C) effects of SfIPPI silencing on ovary development. Significant differences between the treatment and the control groups are indicated by asterisks $\left({ }^{*} p<0.05\right.$ and $\left.{ }^{* *} p<0.01\right)$.

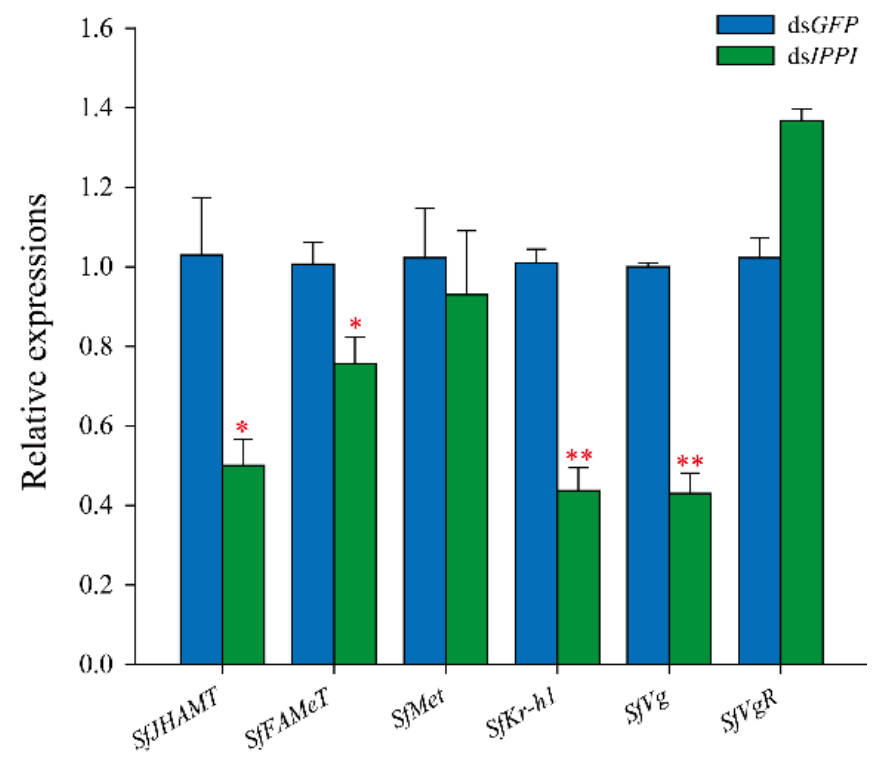

Figure 5. Effect of SfIPPI silencing on the expression of other genes in the juvenile hormone signal transduction pathway. Significance of differences between the treatment group (dsIPPI) and the control group (dsGFP) was determined using the Student $t$-test for independent samples $\left({ }^{*} p<0.05\right.$ and $\left.{ }^{* *} p<0.01\right)$. 


\section{Discussion}

IPPI is a crucial enzyme in the mevalonate pathway in JH synthesis, which catalyzes the conversion of IPP to DMAPP [11] and is crucial for JH biosynthesis. In this study, we successfully cloned the ORF sequence of IPPI from $S$. furcifera using PCR. Bioinformatic analyses revealed that, similar to other insects, SfIPPI encoded a protein sequence containing a typical conserved domain of the Nudix family. Additionally, the phylogenetic analysis showed that SfIPPI was highly similar to L. striatellus and N. lugens.

The expression levels of SfIPPI in different developmental stages and tissues of S. furcifera were detected by qPCR. SfIPPI was expressed and fluctuated in all developmental stages of $S$. furcifera and was highest in $5 \mathrm{~d}$ old adults. SfIPPI expression was detected in all tissues and was highly expressed not only in the integument and head tissues, but also in ovary tissue, suggesting that IPPI is essential for the reproduction of adult $S$. furcifera. The IPPI expression levels were similar to those of other insects. In A. aegypti, the mRNA level of AaIPPI in the corpus allatum was reported to increase $6 \mathrm{~d}$ before eclosion and was highest $24 \mathrm{~h}$ after eclosion, and changes in the pattern of $\mathrm{JH}$ biosynthesis were similar [14]. Furthermore, a study of Dendroctonus armandi revealed that DaIDI expression increased significantly during the adult stage [33]. Similarly, during the development of Leptinotarsa decemlineata, the expression level of LdIDI fluctuated during development, and LdIDI expression was detected in the midgut, Malpighian tube, fat body, epidermis, and other tissues [34].

Many studies have investigated how other genes in the JH signal transduction pathway regulate insect reproduction. For example, after RNAi silencing, the expression of BdHMGR, $B d M e t$, and $B d K r-h 1$ and the ovary development of the female Bactrocera dorsalis was significantly inhibited, and the amount of oviposition was significantly reduced $[35,36]$. In $N$. lugens, after RNAi of JH signaling pathway genes NlTai, NlMet, and NlKr-h1, ovarian development was affected, the pre-oviposition period was prolonged, and the number of oviposited eggs was reduced [37-39]. Similarly, the RNAi inhibition of the FAMeT gene in males or females significantly reduced the number of eggs laid by female Ceratitis capitata [40]. Furthermore, Gijbels et al. [41] reported that female Schistocerca gregaria injected with dsMet could not lay eggs.

All of the above studies have confirmed that JH signal transduction pathway genes are essential for insect reproduction. The inhibition of $\mathrm{JH}$ signal transduction genes by RNAi, subsequently, leads to the inhibition of ovarian development, thereby reducing the amount of oviposition and prolonging preoviposition. However, there are few reports of the regulation of insect reproduction by IPPIs. In this study, we used RNAi technology to analyze the role of the JH synthesis pathway gene SfIPPI in S. furcifera reproduction. Our results showed that the ovarian development of females treated with dsIPPI was significantly inhibited and the number of eggs laid was significantly lower compared to the control group (treated with dsGFP), which was consistent with the function of other genes in the JH synthesis pathway. Targeted silencing of SfJHAMT and SfFAMeT of S. furcifera inhibited the fecundity, ovarian development, and transcription levels of $S f K r-h 1$ and $S f V g$ significantly [42]. These findings suggest that SfIPPI affects the ovarian development of $S$. furcifera and, consequently, the number of eggs laid.

In this study, we showed that $\mathrm{JH}$ synthesis pathway genes are crucial for insect reproduction. Furthermore, SfIPPI silencing significantly inhibited the transcription levels of JH signal transduction pathway-related genes (SfJHAMT, SfFAMeT, and SfKr-h1) and $S f V g$ in $S$. furcifera. Our findings suggest that SfIPPI affects the ovarian development and fecundity of $S$. furcifera by regulating the transcription levels of other genes in the $\mathrm{JH}$ signal transduction pathway and of $S f V g$. Similar results have been observed in the cotton bollworm (Helicoverpa armigera), where the targeted silencing of HMGR expression significantly inhibited female oviposition and $V g$ expression [43]. Thus, the JH biosynthetic pathway gene SfIPPI plays a crucial role in insect ovarian development and reproduction.

These results can provide a theoretical basis for the application of $\mathrm{JH}$ in the green control of rice planthoppers. 


\section{Conclusions}

In summary, we identified SFIPPI in the S. furcifera genome and transcriptome. The bioinformatics analysis revealed a typical conserved domain of the Nudix family, and the phylogenetic analysis showed that SfIPPI had the highest similarity with L. striatellus and N. lugens. The pattern expression analysis indicated that SfIPPI was expressed in all developmental stages and in different tissues of $S$. furcifera, which may be associated with physiological processes. Additionally, SfIPPI silencing significantly inhibited the ovarian development and fecundity of females and significantly inhibited the transcription level of downstream genes in the JH synthesis pathway. Our data could help to clarify the structure, phylogeny, expression models, and biological functions of SfIPPI to further understand the function of $\mathrm{JH}$ biosynthetic pathway genes in insect reproduction and to provide a reference for the prevention and control of agricultural pests in the future.

Author Contributions: M.-F.G. and H.Y. conceived and designed the study; Z.-Y.J. and Q.-H.Z. reared the insects; G.-Y.L. and X.-B.Y. finished the sample preparation; M.-F.G. and C.Z. performed the experiments and analyzed the data; M.-F.G. prepared the manuscript; M.-F.G., C.Z., H.Y., D.-C.J., G.-Y.L. and X.-B.Y. finalized the manuscript. All authors have read and agreed to the published version of the manuscript.

Funding: This work was supported by the National Natural Science Foundation of China (31960537 and 32160635), the Science and Technology Program of Guizhou Province, China ((2020)1Y105), the program of Excellent Innovation Talents in Guizhou Province (20206003), the Guizhou Province Graduate Research Fund YJSCXJH ((2020) 075), and the International Cooperation Base for Insect Evolutionary Biology and Pest Control ((2016) 5802).

Institutional Review Board Statement: Not applicable.

Data Availability Statement: All data are provided within the text.

Acknowledgments: We thank Yue Zhang and Jia-Peng Yang for their assistance with the manuscript revision.

Conflicts of Interest: The authors declare no conflict of interest.

\section{References}

1. Ouyang, Y.C.; Li, S. Modification and application of a high performance liquid chromatography method to separate juvenile hormones and their metabolites. Acta Entomol. Sin. 2003, 3, 282-287.

2. Wigglesworth, V.B. Assays on Rhodnius for juvenile hormone activity. J. Insect Physiol. 1973, 19, 205-211. [CrossRef]

3. Konopova, B.; Jindra, M. Juvenile hormone resistance gene Methoprene-tolerant controls entry into metamorphosis in the beetle Tribolium castaneum. Proc. Natl. Acad. Sci. USA 2007, 104, 10488-10493. [CrossRef] [PubMed]

4. Gao, Q.; Li, B.; Tian, Z.; De, L.A.; Wang, J.L.; Wang, X.P.; Liu, W. Key role of juvenile hormone in controlling reproductive diapause in females of the Asian lady beetle Harmonia axyridis. Pest Manag. Sci. 2022, 78, 193-204. [CrossRef]

5. Gilbert, L.I.; Granger, N.A.; Roe, R.M. The juvenile hormones: Historical facts and speculations on future research directions. Insect Biochem. Mol. Biol. 2000, 30, 617-644. [CrossRef]

6. Luo, W.; Liu, S.; Zhang, W.Q.; Yang, L.; Huang, J.H.; Zhou, S.T.; Feng, Q.L.; Palli, S.R.; Wang, J.; Roth, S.; et al. Juvenile hormone signaling promotes ovulation and maintains egg shape by inducing expression of extracellular matrix genes. Proc. Natl. Acad. Sci. USA 2021, 118, e2104461118. [CrossRef]

7. Rantala, M.J.; Dubovskiy, I.M.; Pölkki, M.; Krama, T.; Contreras-Garduño, J.; Krams, I.A. Effect of juvenile hormone on resistance against entomopathogenic fungus Metarhizium robertsii differs between sexes. J. Fungi 2020, 6, 298. [CrossRef]

8. Zhu, J.; Noriega, F.G. The Role of juvenile hormone in mosquito development and reproduction. Adv. Insect Physiol. 2016, 51, 93-113.

9. Kinjoh, T.; Kaneko, Y.; Itoyama, K.; Mita, K.; Hiruma, K.; Shinoda, T. Control of juvenile hormone biosynthesis in Bombyx mori: Cloning of the enzymes in the mevalonate pathway and assessment of their developmental expression in the corpora allata. Insect Biochem. Mol. Biol. 2007, 37, 808-818. [CrossRef]

10. Bellés, X.; Martín, D.; Piulachs, M.D. The mevalonate pathway and the synthesis of juvenile hormone in insects. Annu. Rev. Entomol. 2005, 50, 181-199. [CrossRef]

11. Goodman, W.G.; Granger, N.A. The Juvenile Hormones. In Comprehensive Molecular Insect Science; Gilbert, L.I., Iatrou, K., Gill, S.S., Eds.; Elsevier: Amsterdam, The Netherlands, 2005; pp. 55-155. 
12. Koyama, T.; Matsubara, M.; Ogura, K. Isoprenoid enzyme systems of silkworm. I. Partial purification of isopentenyl pyrophosphate isomerase, farnesyl pyrophosphate synthetase, and geranylgeranyl pyrophosphate synthetase. J. Biochem. 1985, 98, 449-456 [CrossRef] [PubMed]

13. Sen, S.E.; Tomasello, A.; Grasso, M.; Denton, R.; Macor, J.; Béliveau, C.; Cusson, M.; Crowell, D.N. Cloning, expression and characterization of lepidopteran isopentenyl diphosphate isomerase. Insect Biochem. Mol. Biol. 2012, 42, 739-750. [CrossRef]

14. Diaz, M.E.; Mayoral, J.G.; Priestap, H.; Nouzova, M.; Rivera-Perez, C.; Noriega, F.G. Characterization of an isopentenyl diphosphate isomerase involved in the juvenile hormone pathway in Aedes aegypti. Insect Biochem. Mol. Biol. 2012, 42, 751-757. [CrossRef] [PubMed]

15. Ghaffar, M.B.; Pritchard, J.; Ford-Lloyd, B. Brown planthopper (N. lugens Stål) feeding behavior on rice germplasm as an indicator of resistance. PLoS ONE 2011, 6, e22137. [CrossRef] [PubMed]

16. Luo, X.N.; Zhuo, W.X. The life history of the white-backed planthopper, Sogatella furcifer (Horvath), on its weed host in Fujian Province. J. Plant Prot. 1986, 1, 9-16.

17. Zhang, D.W.; Yu, Y.Y.; Pan, B.Y.; Kang, K.; Zeng, B.P.; Chen, J.; Tang, B. Regulation function of trehalose-6-phosphate synthase genes on chitin synthesis in Sogatella furcifera. Sci. Agric. Sin. 2019, 52, 3357-3366.

18. Zhai, B.P. Rice planthoppers: A China problem under the international perspectives. Chin. J. Appl. Entomol. 2011, 48, 1184-1193.

19. Cheng, J.A. Rice planthopper problems and relevant causes in China. Planthoppers New Threat. Sustain. Intensive Rice Prod. Syst. Asia 2009, 157, 178.

20. Sheng, J.H.; Shang, J.M.; Liu, G.J. Management of the White backed planthopper Sogatella furcifera in China: A Mini review. Chin. J. Rice Sci. 2003, S1, 12-27.

21. Zhou, G.H.; Wen, J.J.; Cai, D.J.; Li, P.; Xu, D.L.; Zhang, S.G. Southern rice black-streaked dwarf virus: A new proposed Fijivirus species in the family Reoviridae. Chin. Sci. Bull. 2008, 53, 3677-3685. [CrossRef]

22. Ruan, Y.W.; Liu, X.X.; Gong, C.W.; Zhang, Y.M.; Shen, L.T.; Ali, H.; Huang, Y.Y.; Wang, X.G. Cloning and functional verification of CYP408A3 and CYP6CS3 related to chlorpyrifos resistance in the Sogatella furcifera (Horváth) (Hemiptera: Delphacidae). Biology 2021, 10, 795. [CrossRef] [PubMed]

23. $\mathrm{Mu}$, X.C.; Zhang, W.; Wang, L.X.; Zhang, S.; Zhang, K.; Gao, C.F.; Wu, S.F. Resistance monitoring and cross-resistance patterns of three rice planthoppers, Nilaparvata lugens, Sogatella furcifera and Laodelphax striatellus to dinotefuran in China. Pestic Biochem. Physiol. 2016, 134, 8-13. [CrossRef]

24. Zhang, X.L.; Liao, X.; Mao, K.K.; Li, J.H.; Wan, H. Insecticide resistance monitoring in field populations of the white-back planthopper, Sogatella furcifera (Hemiptera: Delphacidae) in riceproduction areas of Hubei Province, central China. Acta Entomol. Sin. 2016, 59, 1213-1221.

25. Pradeep, A.R.; Nair, V.S.K. Acquiring vitellogenic competence in the rice pest Nilaparvata lugens Stal: Effects of a juvenile hormone analogue, hydroprene. Int. J. Ind. Ergonom. 2005, 10, 137-141.

26. Lu, K.; Chen, X.; Liu, W.T.; Zhang, X.Y.; Chen, M.X.; Zhou, Q. Nutritional signaling regulates vitellogenin synthesis and egg development through juvenile hormone in Nilaparvata lugens (Stål). Int. J. Mol. Sci. 2016, 17, 269. [CrossRef] [PubMed]

27. Kang, K.; Cai, Y.J.; Zhang, D.W.; Gong, J.; Zhang, W.Q. Effects of nutrition on the growth and reproductive signaling pathways in the brown planthopper, Nilaparvata lugens (Hemiptera: Delphacidae). Acta Entomol. Sin. 2021, 64, 1377-1387.

28. Lu, K.; Zhou, J.; Chen, X.; Li, W.R.; Li, Y.; Cheng, Y.B.; Yan, J.; You, K.K.; Yuan, Z.N.; Zhou, Q. Deficiency of brummer impaires lipid mobilization and JH-mediated vitellogenesis in the brown planthopper, Nilaparvata lugens. Front. Physiol. 2018, 9, 1535. [CrossRef] [PubMed]

29. Wang, L.; Tang, N.; Gao, X.L.; Chang, Z.X.; Zhang, L.Q.; Zhou, G.H.; Guo, D.Y.; Zeng, Z.; Li, W.J.; Akinyemi, I.A.; et al. Genome sequence of a rice pest, the white-backed planthopper (Sogatella furcifera). GigaScience 2017, 6, giw004.

30. Zhou, C.; Yang, H.; Wang, Z.; Long, G.Y.; Jin, D.C. Comparative transcriptome analysis of Sogatella furcifera (Horvath) exposed to different insecticides. Sci. Rep. 2018, 8, 8773. [CrossRef]

31. Tamura, K.; Stecher, G.; Peterson, D.; Filipski, A.; Kumar, S. MEGA6: Molecular evolutionary genetics analysis version 6.0. Mol. Biol. Evol 2013, 30, 2725-2729. [CrossRef]

32. Livak, K.J.; Schmittgen, T.D. Analysis of relative gene expression data using real-time quantitative PCR and the $2-\Delta \Delta C T$ method. Methods 2001, 25, 402-408. [CrossRef]

33. Wang, Y.Y.; Yu, J.M.; Dai, L.L.; Cheng, H. Cloning and expression of two mevalonate pathway genes of Dendroctonus armandi. J. Northwest For. Univ. 2020, 35, 140-149.

34. Li, Q.; Meng, Q.W.; Lü, F.G.; Guo, W.C.; Li, G.Q. Identification of ten mevalonate enzyme-encoding genes and their expression in response to juvenile hormone levels in Leptinotarsa decemlineata (Say). Gene 2016, 584, 136-147. [CrossRef]

35. Yang, W.J.; Xu, K.K.; Dou, W.; Li, C.; Wang, J.J. 3-Hydroxy-3-methyl glutaryl coenzyme A reductase is required for ovarian development in the oriental fruit fly Bactrocera dorsalis (Hendel). J. Asia Pac. Entomol. 2018, 21, 1071-1078. [CrossRef]

36. Yue, Y.; Yang, R.L.; Wang, W.P.; Zhou, Q.H.; Chen, E.H.; Yuan, G.R.; Wang, J.J.; Dou, W. Involvement of Met and Kr-h1 in JH-Mediated Reproduction of Female Bactrocera dorsalis (Hendel). Front. Physiol. 2018, 9, 482. [CrossRef]

37. Li, Y.; Gao, H.; Zhang, Y.; Lin, X. Role of the transcription factor Taiman in moulting and ovarian development of Nilaparvata lugens. Entomol. Gen. 2021, 41, 169-177. [CrossRef]

38. Lin, X.; Yao, Y.; Wang, B. Methoprene-tolerant (Met) and Krüpple-homologue $1(K r-h 1)$ are required for ovariole development and egg maturation in the brown plant hopper. Sci. Rep. 2015, 5, 18064. [CrossRef] [PubMed] 
39. Jiang, J.; Xu, Y.; Lin, X. Role of Broad-Complex (Br) and Krüppel homolog $1(K r-h 1)$ in the ovary development of Nilaparvata lugens. Front. Physiol. 2017, 8, 1013. [CrossRef]

40. Vannini, L.; Ciolfi, S.; Dallai, R.; Frati, F.; Hoffmann, K.H.; Meyering-Vos, M. Putative-farnesoic acid O-methyltransferase (FAMeT) in medfly reproduction. Arch. Insect Biochem. Physiol. 2010, 75, 92-106. [CrossRef]

41. Gijbels, M.; Lenaerts, C.; Vanden Broeck, J.V.; Marchal, E. Juvenile hormone receptor Met is essential for ovarian maturation in the desert locust, Schistocerca gregaria. Sci. Rep. 2019, 9, 10797. [CrossRef]

42. Zhou, C.; Yang, X.B.; Yang, H.; Gong, M.F.; Long, G.Y.; Jin, D.C. Role of SfJHAMT and SfFAMeT in the reproductive regulation of Sogatella furcifera and its expression under insecticide stress. Pestic Biochem. Phys. 2021, 173, 104779. [CrossRef] [PubMed]

43. Wang, Z.; Dong, Y.; Desneux, N.; Niu, C. RNAi silencing of the HaHMG-CoA reductase gene inhibits oviposition in the Helicoverpa armigera cotton bollworm. PLoS ONE 2013, 8, e67732. [CrossRef] [PubMed] 